\title{
Venous Thromboembolism and Risk of Cancer in Patients with Dementia: A Danish Population-Based Cohort Study
}

\author{
Cecilia H. Fuglsang ${ }^{\mathrm{a}, *}$, David Nagy ${ }^{\mathrm{a}}$, Frederikke S. Troelsen ${ }^{\mathrm{a}}$, Dora K. Farkas ${ }^{\mathrm{a}}$, \\ Victor W. Henderson ${ }^{\mathrm{a}, \mathrm{b}, \mathrm{c}}$ and Henrik T. Sørensen ${ }^{\mathrm{a}, \mathrm{d}}$ \\ ${ }^{a}$ Department of Clinical Epidemiology, Aarhus University/Aarhus University Hospital, Aarhus, Denmark \\ ${ }^{\mathrm{b}}$ Department of Epidemiology \& Population Health, Stanford University, Stanford, CA, USA \\ ${ }^{\mathrm{c}}$ Department of Neurology \& Neurological Sciences, Stanford University, Stanford, CA, USA \\ ${ }^{\mathrm{d}}$ Center for Population Health Sciences, Stanford, CA, USA
}

Handling Associate Editor: M. Arfan Ikram

Accepted 26 May 2021

Pre-press 26 June 2021

\begin{abstract}
.
Background: Venous thromboembolism (VTE) may be the first manifestation of occult cancer. Dementia has been linked to reduced cancer risk.

Objective: We examined the risk of cancer following VTE in people with dementia in comparison to the risk in the general population.

Methods: We conducted a population-based Danish registry-based cohort study following patients with a first-time VTE and a previous or concurrent diagnosis of dementia during the period 1 April 1996 - 31 December 2017. We followed the study participants from date of VTE until diagnosis of cancer, death, emigration, or end of study period, whichever came first. The absolute risk of cancer within one year after VTE was computed, treating death as a competing risk. We calculated gender, age, and calendar-period standardized incidence ratios (SIRs) of cancer based on national cancer rates.

Results: We followed 3,552 people with dementia and VTE for a median of 1.3 years. Within the first year after VTE, they had a 90\% increased risk of cancer in comparison with the general population [SIR: 1.9 (95\% confidence interval: 1.6-2.4)]. During subsequent follow-up years, the SIR fell to 0.7 (95\% confidence interval: 0.5-0.8). Findings for Alzheimer's disease and VTE were similar.

Conclusion: People with dementia have an increased risk of a cancer diagnosis during the first year following VTE, perhaps related to increased surveillance, and a lower risk thereafter. Overall risk is similar to that of the general population.
\end{abstract}

Keywords: Alzheimer's disease, cohort study, dementia, epidemiology, neoplasms, venous thromboembolism

\footnotetext{
${ }^{*}$ Correspondence to: Cecilia H. Fuglsang, MD, Department of Clinical Epidemiology, Aarhus University, Olof Palmes Allé 43-45, Aarhus N, Denmark. Tel.: +4587168486; E-mail: chfn@clin.au.dk.
}

\section{INTRODUCTION}

The association between cancer and the clotting system has been recognized for more than 150 years, since Armand Trousseau reported a high rate of venous thrombosis in patients with a visceral malignancy [1]. This association is bi-directional. Twenty percent of Danish venous thrombosis patients have a 
diagnosed cancer at time of their venous thromboembolism (VTE) diagnosis [2]. At the same time, cancer patients have a 5- to 10-fold increased risk of VTE, with a particularly high risk in the period just after the cancer diagnosis [3]. There is strong evidence that VTE may be the first manifestation of occult cancer $[4,5]$. In their 2008 systematic review, Carrier et al. [6] reported a $6.1 \%$ rate of undiagnosed cancer at the time of a first thromboembolic event.

One Swedish study examined the association between VTE and subsequent cancer risk in people with dementia. In 2017, Sandén et al. [7] reported predictors of cancer among VTE patients. Among 100 people with dementia and a first-time VTE, they found a one-year hazard ratio of cancer of 0.30 [95\% confidence interval (CI) 0.10-0.95] compared to patients with VTE alone. A number of studies have reported a reduced risk of cancer among dementia patients in general [8-10], but it is not clear whether people with dementia face the same cancer risk after VTE. For this reason, we assessed the risk of cancer after a first-time diagnosis of VTE, including both deep venous thrombosis (DVT) or pulmonary embolism (PE), in Danish dementia patients using nationwide, population-based healthcare data.

\section{METHODS}

\section{Setting}

The entire Danish population is covered by a taxfunded healthcare system [11]. Residents' contacts with the health care system are recorded in national registries together with their unique personal identification number [Civil Personal Register number (CPR number)] [12]. Data in medical and administrative registries can be linked by means of the CPR number [11]. Using these registries, we conducted a nationwide population-based cohort study over the period 1 April 1996 - 31 December 2017. Our study population consisted of all Danish residents (cumulatively numbering approximately 6.9 million individuals during the nearly 22 -year period) [12].

\section{Data sources and variables}

Since 1977, admissions to non-psychiatric hospitals in Denmark have been registered in the Danish National Patient Registry (DNPR). As of 1978, coverage has been complete [13]. The Danish Central
Psychiatric Registry [14], containing data on all psychiatric hospital admissions, was integrated into the DNPR in 1995. That same year, data on all outpatient and emergency room contacts at non-psychiatric and psychiatric hospitals began to be collected. At each hospital contact, administrative and personal data are collected, including the patient's CPR number, dates of admission and discharge, type of contact (outpatient clinic, emergency room, or inpatient), contact reason, one primary diagnosis, and up to $19 \mathrm{sec}-$ ondary diagnoses. Since 1994, diagnoses have been coded according to the World Health Organization's International Classification of Diseases, Tenth Revision (ICD-10). Previous to this, diagnoses were coded using ICD-8. Since 1996, surgeries have been coded according to a Danish version of the Nordic MedicoStatistical Committee (NOMESCO) Classification of Surgical Procedures [13].

All malignant neoplasms in Denmark are recorded in the Danish Cancer Registry (DCR). Since 2004, cancer type has been registered according to ICD10 coding. Earlier diagnoses, going back to 1978, have been converted to ICD-10. Since 2004, solid tumors have been categorized according to the Union for International Cancer Control Tumor, Nodes, and Metastases (TNM) classification system. Up to that time, staging was recorded using the International Federation of Gynecology and Obstetrics (FIGO) classification, Dukes classification, or a registrar's own classification system [15].

We used the DNPR to identify all patients 50 years or older with a first-time VTE diagnosis and a previous or concurrent diagnosis of dementia (both primary and secondary diagnoses). We excluded emergency room diagnoses, as they have low validity, i.e., VTE is confirmed in only $50 \%$ of patients for whom the diagnosis is suspected $[16,17]$.

VTE was defined as a diagnosis of either a DVT or a PE. The primary outcome was occurrence of any type of cancer (not including non-melanoma skin cancer), as registered in the DCR. Patients with a cancer diagnosis in the DCR before the date of VTE diagnosis were not included in the study. In this approach, only incident cases of cancer were included as outcome.

The DNPR was used to obtain information on factors that provoke VTE and on the burden of comorbidity at baseline. Pregnancy, surgery, trauma, or fractures up to three months prior to a VTE were identified as provoking factors. We assessed comorbidity using the Charlson Comorbidity Index (CCI) [18], including all $\mathrm{CCI}$ conditions occurring before and on the VTE date, except dementia and cancer. Cohort 


\begin{tabular}{|c|c|c|c|c|c|c|}
\hline Category & $\begin{array}{c}\mathrm{FU}^{+} \\
\text {years }\end{array}$ & $\begin{array}{r}\text { AR } \\
\%(95 \% \text { Cl) }\end{array}$ & $\underset{\text { count }}{\text { OBS }^{+}}$ & $\begin{array}{r}\text { SIR } \\
(95 \% \text { CI) }\end{array}$ & & \\
\hline All cancers & $0-1$ & $2.8(2.3-3.4)$ & $\begin{array}{l}97 \\
79\end{array}$ & $1.9(1.6-2.4)$ & 吅 & $\square$ \\
\hline Dementia & & & & & & \\
\hline Alzheimer's disease & $0-1$ & $2.6(1.8-3.7)$ & 29 & $1.7(1.2-2.5)$ & & $\mapsto \square$ \\
\hline Gender & $1+$ & & 14 & $0.4(0.2-0.7)$ & $\square-$ & \\
\hline Female & $0-1$ & $2.4(1.8-3.1)$ & 53 & $1.9(1.4-2.4)$ & & $\mapsto \square$ \\
\hline & $1+$ & & 39 & $0.6(0.4-0.8)$ & $\square$ & \\
\hline Male & $0-1$ & $3.5(2.6-4.6)$ & 44 & $2.0(1.5-2.7)$ & & $\longmapsto \square$ \\
\hline & $1+$ & & 40 & $0.7(0.5-1.0)$ & 叶 & \\
\hline Age at VTE diagnosis & & & & & & \\
\hline $50-69$ years & $0-1$ & $2.6(1.4-4.4)$ & 12 & $2.5(1.3-4.4)$ & & $\square$ \\
\hline & $1+$ & & 29 & $1.1(0.7-1.6)$ & $\vdash$ & $\rightarrow$ \\
\hline $70-74$ years & $0-1$ & $4.1(2.5-6.5)$ & 16 & $2.6(1.5-4.2)$ & & $\square$ \\
\hline & $1+$ & & 12 & $0.6(0.3-1.0)$ & $\mapsto \square$ & \\
\hline $75-79$ years & $0-1$ & $3.9(2.5-5.7)$ & 23 & $2.3(1.4-3.4)$ & & $\square$ \\
\hline & $1+$ & & 24 & $1.0(0.7-1.5)$ & $\rightarrow$ & 七 \\
\hline $80-84$ years & $0-1$ & $2.6(1.7-3.9)$ & 21 & $1.5(1.0-2.4)$ & & $\square$ \\
\hline & $1+$ & & 6 & $0.2(0.1-0.5)$ & $\square-$ & \\
\hline $85-89$ years & $0-1$ & $2.4(1.5-3.6)$ & 19 & $1.7(1.0-2.7)$ & & $\square$ \\
\hline & $1+$ & & $-^{*}$ & $0.3(0.1-0.7)$ & $\square$ & \\
\hline+90 years & $0-1$ & $1.3(0.5-2.7)$ & 6 & $1.4(0.5-3.0)$ & ط & $\square$ \\
\hline & $1+$ & & $<5$ & $0.4(0.1-1.4)$ & - & $\longrightarrow$ \\
\hline Type of VTE & & & & & & \\
\hline Deep vein thrombosis & $0-1$ & $2.5(1.9-3.3)$ & 52 & $1.6(1.2-2.0)$ & & $1-\square-1$ \\
\hline & $1+$ & & 52 & $0.6(0.5-0.8)$ & 口. & \\
\hline Pulmonary embolism & $0-1$ & $3.1(2.3-4.1)$ & 45 & $2.7(2.0-3.6)$ & & $\longmapsto \square$ \\
\hline & $1+$ & & 27 & $0.7(0.5-1.1)$ & 叶 & \\
\hline Charlson Comorbidit & & & & & & \\
\hline Low & $0-1$ & $2.5(1.8-3.4)$ & 36 & $1.7(1.2-2.3)$ & & $\longmapsto$ \\
\hline & $1+$ & & 33 & $0.6(0.4-0.8)$ & $\square$ & \\
\hline Medium & $0-1$ & $2.7(2.0-3.6)$ & 40 & $1.9(1.4-2.6)$ & & $\longmapsto$ \\
\hline & $1+$ & & 28 & $0.6(0.4-0.8)$ & $\square$ & \\
\hline High & $0-1$ & $3.6(2.3-5.3)$ & 21 & $2.6(1.6-4.0)$ & & $\longmapsto$ \\
\hline & $1+$ & & 18 & $1.3(0.8-2.0)$ & $\theta_{1}$ & $\square$ \\
\hline VTE provoking fac & & & & & & \\
\hline No & $0-1$ & $3.1(2.5-3.8)$ & 83 & $2.1(1.7-2.6)$ & & $\mapsto$ \\
\hline & $1+$ & & 56 & $0.6(0.4-0.8)$ & $\square$ & \\
\hline Yes & $0-1$ & $1.8(1.0-2.9)$ & 14 & $1.4(0.7-2.3)$ & $\mapsto$ & $\square$ \\
\hline & $1+$ & & 23 & $0.9(0.6-1.3)$ & 15 & \\
\hline Cancer stage & & & & & & \\
\hline Localized & $0-1$ & $0.5(0.3-0.7)$ & 16 & $1.1(0.6-1.7)$ & $\mapsto$ & ط— \\
\hline & $1+$ & & 22 & $0.6(0.4-0.9)$ & $\square-1$ & \\
\hline Non-Localized & $0-1$ & $1.0(0.7-1.4)$ & 36 & $2.1(1.5-3.0)$ & & $\longmapsto \square$ \\
\hline & $1+$ & & 23 & $0.6(0.4-0.9)$ & $\square-$ & \\
\hline Unknown & $0-1$ & $1.3(1.0-1.7)$ & 45 & $2.5(1.8-3.3)$ & & $\longmapsto \square$ \\
\hline & $1+$ & & 34 & $0.7(0.5-1.1)$ & $\square$ & \\
\hline & & & & & 00.51 & 11.522 .533 .544 .5 \\
\hline
\end{tabular}

Fig. 1. Risk of cancer following venous thromboembolism (VTE) among people with dementia. First year absolute risk (AR). First and subsequent years standardized incidence ratios (SIRs). *Due to Danish legislation, we report no numbers for groups with less than five observations. Accordingly, observed numbers in subsequent years were not reported for age group 85-89, as it thus would be possible to calculate observed numbers for the age group of $90+$ years. ${ }^{\dagger} \mathrm{FU}$, Follow-up; OBS, observations. ${ }^{\ddagger}$ Charlson Comorbidity Index Score at VTE diagnosis; low $=0$, medium: $1-2$, high $=3$ or more.

members were divided into three CCI categories: low (no comorbidities) $=\mathrm{CCI}$ score of 0 , medium $=\mathrm{CCI}$ score of $1-2$, or high $=\mathrm{CCI}$ score of 3 or more.
Cancers were categorized according to presence of metastases, in order to assess potential detection bias-as described in the study by Ording et al. 
[8]. If patients or physicians underreport symptoms of cancer due to the patient's dementia, it is likely that any cancer is diagnosed at a more advanced stage. We used the DCR to obtain information on tumor stage and categorized tumors as localized, non-localized, or unknown. Non-solid tumors were categorized together with tumors of unknown stage.

All relevant ICD-8, ICD-10, and surgery codes are provided in Supplementary Tables 1 and 2. Codes used to categorize tumor stage are presented in Supplementary Table 3.

\section{Statistical analyses}

Patients were described at baseline according to gender, age group (50-69, 70-74, 75-79, 80-84, 85-89 and +90 years), dementia diagnosis (Alzheimer's disease or any other dementia), CCI score, presence of provoking factors within three months prior to VTE, and type of VTE (DVT or PE). If a patient had diagnoses of both PE and DVT on the same date, the patient was categorized as having a PE only. Patients were followed from the date of VTE occurrence until a cancer diagnosis (not including non-melanoma skin cancer), death, emigration, or administrative end of follow-up (31 December 2017), whichever came first. Sørensen et al. [5] reported that the risk of cancer following VTE in the general population became constant after approximately one year. We therefore divided the follow-up into two periods: the first year after VTE diagnosis and subsequent follow-up years. The risk for the overall period of follow-up was calculated as well.

We computed absolute risks using Aalen-Johansen estimator of the cumulative incidence function during the first follow-up period, treating death as a competing risk. Thus, patients were censored at death in order to prevent underestimating absolute risk of cancer.

We used standardized incidence ratios (SIRs) as a measure of the relative risk of cancer following VTE in people with dementia, presenting the ratio between the observed and the expected number of cancer cases for all follow-up periods. The expected number was calculated based on Danish national incidence rates of first-time diagnoses of cancer by gender, age, and year of diagnosis in 5 year intervals [19].

We stratified both cumulative incidences and SIRs according to gender, age group, CCI score, type of VTE, presence of a provoking factor, and cancer stage. Assuming a Poisson distribution, we calculated 95\% CIs for each stratified category. Byar's approximation was used for categories with 10 or more observed cancers. For fewer than 10 observed cancers, exact $95 \%$ CIs were used [20].

As required by Danish legislation, we report no numbers for groups with fewer than five observations.

All statistical analyses were performed using version 9.4 of the SAS statistical software package. Figures were created using statistical software R version 4.04 (2021-02-15). The study was reported to the Danish Data Protection Agency by Aarhus University (record number 2016-051-000001/811) and no further approvals were required according to Danish law.

\section{RESULTS}

\section{Descriptive data}

During the study period, we identified 3,552 patients with a first-time VTE and a previous or concurrent diagnosis of dementia. Construction of this cohort is illustrated in Supplementary Figure 1. The median age at VTE diagnosis was 82 years [interquartile range (IQR): 75-87 years] and 64\% were female (Table 1). Thirty-two percent of patients had Alzheimer's disease. The most common VTE type was DVT. In our study population, almost $60 \%$ had at least one of the comorbidities included in the CCI. In $22 \%$ of patients, we identified at least one VTE provoking factor.

We followed the cohort for a total of 8,096 personyears. The median follow-up time was 1.3 years (IQR: $0.2-3.3$ years).

\section{Cancer}

\section{First year of follow-up}

During follow-up, 176 cancers were identified among the VTE patients with dementia. Of these, 97 cancers were diagnosed within the first year after VTE diagnosis (Fig. 1). The absolute risk of cancer within the first year was thus $2.8 \%$ (95\% CI: 2.3-3.4\%). This corresponded to an SIR of 1.9 (95\% CI: 1.6-2.4). Patients with Alzheimer's disease had an absolute risk of $2.6 \%$ (95\% CI: $1.8-3.7 \%$ ) and an SIR of 1.7 (95\% CI:1.2-2.5).

Both the absolute risk of cancer and the SIR were higher for patients with a PE than for patients with a DVT.

Gender did not have an effect on the SIR. Presence of a VTE provoking factor, a low-medium burden of 
Table 1

Characteristics of patients with first-time venous thromboembolism (VTE) and a previous or concurrent diagnosis of dementia during the period 1 April 1996 - 31 December 2017

\begin{tabular}{|c|c|}
\hline & $n(\%)$ \\
\hline Dementia, all & $3,552(100)$ \\
\hline Alzheimer's disease & $1,137(32.0)$ \\
\hline \multicolumn{2}{|l|}{ Gender } \\
\hline Female & $2,274(64.0)$ \\
\hline Male & $1,278(36.0)$ \\
\hline \multicolumn{2}{|l|}{ Age at VTE diagnosis } \\
\hline $\begin{array}{l}\text { Median age at VTE diagnosis in } \\
\text { years (IQR*) }\end{array}$ & $82.0(75.3-87.3)$ \\
\hline $50-69$ y & $461(13.0)$ \\
\hline $70-74 \mathrm{y}$ & $391(11.0)$ \\
\hline $75-79 y$ & $603(17.0)$ \\
\hline $80-84$ y & $814(22.9)$ \\
\hline $85-89 y$ & $811(22.8)$ \\
\hline$+90 y$ & $472(13.3)$ \\
\hline \multicolumn{2}{|l|}{ Type of VTE } \\
\hline Deep vein thrombosis & $2,096(59.0)$ \\
\hline Pulmonary embolism & $1,456(41.0)$ \\
\hline \multicolumn{2}{|l|}{ Charlson Comorbidity Index burden ${ }^{\dagger}$} \\
\hline Low & $1,450(40.8)$ \\
\hline Medium & $1,504(42.4)$ \\
\hline High & $598(16.8)$ \\
\hline \multicolumn{2}{|l|}{ VTE provoking factor } \\
\hline No & $2,755(77.6)$ \\
\hline Yes & $797(22.4)$ \\
\hline \multicolumn{2}{|l|}{ VTE provoking factor by condition } \\
\hline None & $2,755(77.6)$ \\
\hline Trauma/fracture only & $328(9.2)$ \\
\hline Surgery only & $192(5.4)$ \\
\hline Pregnancy only & $0(0.0)$ \\
\hline Multiple factors & $277(7.8)$ \\
\hline Follow-up, median years after VTE (IQR)* & $1.3(0.2-3.3)$ \\
\hline
\end{tabular}

${ }^{*} \mathrm{IQR}=$ Interquartile range. ${ }^{\dagger}$ Charlson Comorbidity Index score; low $=0$, medium $=1-2$, high $=3$ or more. ${ }^{\ddagger}$ Pregnancy, surgery, trauma, or fracture up to three months prior to VTE.

comorbidity, older age, and presence of a localized cancer were associated with lower SIRs.

The most common cancers recorded during the first year of follow-up were cancers of the lung, colon, prostate, kidney, and urinary bladder, together with metastases and non-specified cancer in lymph nodes (Fig. 2).

\section{Subsequent follow-up years}

During subsequent years of follow-up, 79 cancers were observed and the SIR fell to 0.7 (95\% CI: 0.5-0.8) (Fig. 1). SIR among patients with Alzheimer's disease was 0.4 (95\% CI: 0.2-0.7). The estimated SIRs were similar across gender, type of VTE, and stage of cancer. Absence of a provoking factor for VTE, older age, and a low-medium burden of comorbidity was associated with a lower SIR.
The most common cancer diagnoses after the first year of follow-up were cancers of the colon, lung, breast, prostate, rectum, and non-Hodgkin's lymphoma as well as malignant melanoma (Fig. 2).

\section{Overall follow-up}

The absolute risk of cancer during the total 22-year follow-up period was $6.0 \%$ (95\% CI: 5.2-7.06.8-9.2), representing an SIR of 1.0 (95\% CI: $0.9-1.2)$. For Alzheimer's disease, the SIR was 0.8 (95\% CI: 0.6-1.1).

\section{DISCUSSION}

In this nationwide Danish cohort study of dementia patients, an incident VTE was associated with an approximately $3 \%$ absolute risk of a cancer diagnosis during the first year of follow-up. This corresponded to a $90 \%$ increased relative risk of cancer compared to members of the general population of same age, gender, and calendar period. In the subsequent years of follow-up, the risk of cancer among dementia patients with VTE was 30\% lower than in the general population. For the entire follow-up period, the cancer risk in people with dementia was about the same as in the general population.

Previous studies have reported an association between VTE and cancer for the general population, albeit with risk estimates greater than for our dementia cohort [4, 21]. However, our study findings are consistent with those of Sandén et al. [7] who reported a 0.30 (95\% CI: $0.10-0.95$ ) hazard ratio of cancer within the first year after VTE for patients with dementia compared to the VTE in the population without dementia.

For follow-up periods beyond one year, previous studies reported SIRs of approximately one [4, 5]. In our dementia cohort, the SIR dropped to 0.7. This SIR is similar to the a priori risk of cancer among Danish dementia patients, as reported by Ording et al. [8] in 2019. Looking at the entire follow-up period, the SIR was one.

One interpretation of our findings is that overall cancer risk in people with dementia and a first-time VTE is not increased. Rather, it is similar to that of the general population. The association between VTE and cancer in the general population is well recognized, and heightened surveillance soon after VTE in people with dementia could facilitate the detection of occult cancer during the first year of follow-up that might not have become evident until later. Thus, 


\begin{tabular}{|c|c|c|c|c|c|}
\hline Type of Cancer* & $\begin{array}{r}\mathrm{FU}^{+} \\
\text {years }\end{array}$ & $\begin{array}{r}\text { AR } \\
\%(95 \% \mathrm{Cl})\end{array}$ & $\begin{array}{l}\text { OBS }^{+} \\
\text {count }\end{array}$ & $\begin{array}{r}\text { SIR } \\
(95 \% \mathrm{CI})\end{array}$ & \\
\hline Breast & $1+$ & & 9 & $0.6(0.3-1.2)$ & \\
\hline \multirow[t]{2}{*}{ Colon } & $0-1$ & $0.3(0.2-0.6)$ & 12 & $1.9(1.0-3.4)$ & \\
\hline & $1+$ & & 10 & $0.7(0.3-1.3)$ & \\
\hline Kidney & $0-1$ & $0.1(0.1-0.3)$ & 5 & $5.8(1.9-13.4)$ & \\
\hline \multirow[t]{2}{*}{ Lung } & $0-1$ & $0.5(0.3-0.8)$ & 18 & $2.8(1.7-4.4)$ & $\exists$ \\
\hline & $1+$ & & 11 & $0.7(0.4-1.3)$ & \\
\hline Malignant melanoma & $1+$ & & 5 & $1.1(0.3-2.5)$ & \\
\hline Non-Hodgkin's lymphoma & $1+$ & & 5 & $0.9(0.3-2.1)$ & \\
\hline \multirow[t]{2}{*}{ Prostate } & $0-1$ & $0.2(0.1-0.4)$ & 8 & $1.4(0.6-2.7)$ & \\
\hline & $1+$ & & 6 & $0.4(0.2-0.9)$ & \\
\hline Urinary bladder & $0-1$ & $0.2(0.1-0.4)$ & 6 & $1.8(0.7-4.0)$ & \\
\hline \multirow{3}{*}{$\begin{array}{l}\text { Rectum } \\
\text { Metastases and non-specified } \\
\text { cancer in lymph nodes }\end{array}$} & $1+$ & & 5 & $0.8(0.3-2.0)$ & \\
\hline & $0-1$ & $0.3(0.2-0.5)$ & 11 & $6.3(3.2-11.3)$ & 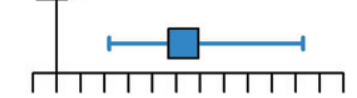 \\
\hline & & & & & 01234567891113 \\
\hline
\end{tabular}

Fig. 2. Risk of cancer following venous thromboembolism (VTE) among people with dementia according to cancer type. First year absolute risk (AR). First and subsequent years standardized incidence ratios (SIRs). ${ }^{*}$ Only sites with 5 or more recorded cancers are presented. ${ }^{\dagger}$ FU, Follow-up; OBS, observations.

increased surveillance after VTE is offset by lower SIRs thereafter.

It is possible that observed SIRs in the Danish population underestimate cancer risk in people with dementia. Within the first year after VTE, the SIR was larger for a PE, a more serious condition, than for a DVT, where the need for less intensive management might reduce the incidental detection of cancer. Of note, lung cancer was the most common cancer detected during the first year after VTE. Also, during the first year after VTE, SIRs were somewhat lower for localized cancer than for metastatic cancer. This result could be due to fewer diagnostic procedures performed after VTE in people with dementia, allowing some localized cancers to remain undetected during this time. The inference is that cancer risk might indeed be increased after VTE, as reported in other populations $[4,5]$.

Overall, influence of VTE on risk of cancer diagnosis seems less pronounced among people with dementia than in other populations. Previous studies have proposed an inverse link between cancer and dementia [8-10]. Papers discussing this matter suggest this could be due to potential surveillance or survival bias. A biologic inverse association is, however, possible. Cancer arises through cell proliferation, contrary to dementia which involves cell death. A biologic underlying mechanism behind the relation may include immunological, genetic, or environmental factors [22, 23]. Nonetheless, it appears people with dementia have an increased risk of cancer diagnosis during the first year after VTE, and a lower risk thereafter. As VTE for some time has been regarded as a marker of malignancy, this finding seems reasonable.

If we consider VTE a potential first manifestation of an undiagnosed cancer among dementia patients, screening for occult cancer could decrease cancerrelated mortality. However, results from randomized trials in the general population do not support the effectiveness of extensive screening on mortality or early cancer detection [24-26]. In our setting, 3,552 patients would have to be screened in order to detect the 46 excess cancers identified during the first year of follow-up. The cost-effectiveness of extensive screening of this predominantly older patient group would probably be low. At the same time, diagnostic procedures may have side-effects and cause both 
physical and psychological distress, especially for those with severe dementia. However, a diagnosis can ensure treatment, if not only alleviating symptoms, which might improve quality of life. The more pragmatic approach of screening based on symptoms of cancer, expected remaining life-years and potential for increasing quality of life seems warranted.

\section{Strengths and limitations}

Strengths of our study are its design and the validity of the registry-based codes. This study had a population-based design with use of nationwide registries, which allowed for complete follow-up. The validity of the codes of dementia, VTE, and cancer in the Danish registries is generally high. After excluding emergency room diagnoses, as we did in our study, the positive predictive value (PPV) of the diagnosis of a first-time VTE in the DNPR is $88 \%$ [27]. Prior research has shown overall PPVs of $95 \%-98 \%$ in the DCR [28]. For dementia, a PPV of $86 \%$ has been reported [29].

Using SIRs as risk estimates implies that the exposed group is included in the comparison cohort [30]. As the exposure of both dementia and VTE is rare, any bias due to this method would likely be small. The main concern is, as mentioned above, the possibility of surveillance bias.

\section{CONCLUSIONS}

Our findings indicate that people with Alzheimer's disease and other forms of dementia face increased risk of a cancer diagnosis during the first year after VTE. The magnitude of this risk may be underestimated due to limited work-up for cancer among dementia patients with VTE. During subsequent follow-up years, cancer risk was reduced compared to the general population, and overall risk was similar to that of the general population. We predicted increased cancer risk in people with dementia and VTE, and surveillance bias in people with dementia could account for lower than expected overall risk.

\section{ACKNOWLEDGMENTS}

This work was supported by the Lundbeck Foundation (grant number R248-2017-521). Funding sources had no involvement in study design, collection, analysis, or interpretation of data, writing the report, or in the decision to submit the article for publication.
Authors' disclosures available online (https:// www.j-alz.com/manuscript-disclosures/20-1530r2).

\section{SUPPLEMENTARY MATERIAL}

The supplementary material is available in the electronic version of this article: https://dx.doi. org/10.3233/JAD-201530.

\section{REFERENCES}

[1] Trousseau A (1865) Phlegmasia alba dolens. Clinique Médicale de l'Hôtel-Dieu de Paris, 2nd edition, J-B Ballière et Fils, Paris, pp. 654-712.

[2] Sørensen HT, Horvath-Puho E, Søgaard KK, Christensen S, Johnsen SP, Thomsen RW, Prandoni P, Baron JA (2009) Arterial cardiovascular events, statins, low-dose aspirin and subsequent risk of venous thromboembolism: A populationbased case-control study. J Thromb Haemost 7, 521-528.

[3] Cronin-Fenton DP, Søndergaard F, Pedersen LA, Fryzek JP, Cetin K, Acquavella J, Baron JA, Sørensen HT (2010) Hospitalisation for venous thromboembolism in cancer patients and the general population: A population-based cohort study in Denmark, 1997-2006. Br J Cancer 103, 947-953.

[4] Baron JA, Gridley G, Weiderpass E, Nyrén O, Linet M (1998) Venous thromboembolism and cancer. Lancet 351, 1077-1080.

[5] Sørensen HT, Mellemkjaer L, Steffensen FH, Olsen JH, Nielsen GL (1998) The risk of a diagnosis of cancer after primary deep venous thrombosis or pulmonary embolism. N Engl J Med 338, 1169-1173.

[6] Carrier M, Le Gal G, Wells PS, Fergusson D, Ramsay T, Rodger MA (2008) Systematic review: The Trousseau syndrome revisited: Should we screen extensively for cancer in patients with venous thromboembolism? Ann Intern Med 149, 323-333.

[7] Sandén P, Svensson PJ, Själander A (2017) Venous thromboembolism and cancer risk. J Thromb Thrombolysis 43, 68-73.

[8] Ording AG, Veres K, Horváth-Puhó E, Glymour MM, Rørth M, Henderson VW, Sørensen HT (2019) Alzheimer's and Parkinson's diseases and the risk of cancer: A cohort study. J Alzheimers Dis 72, 1269-1277.

[9] Attner B, Lithman T, Noreen D, Olsson H (2010) Low cancer rates among patients with dementia in a populationbased register study in Sweden. Dement Geriatr Cogn Disord 30, 39-42.

[10] Driver JA, Beiser A, Au R, Kreger BE, Splansky GL, Kurth T, Kiel DP, Lu KP, Seshadri S, Wolf PA (2012) Inverse association between cancer and Alzheimer's disease: Results from the Framingham Heart Study. BMJ 344, e1442.

[11] Schmidt M, Schmidt SAJ, Adelborg K, Sundbøll J, Laugesen K, Ehrenstein V, Sørensen HT (2019) The Danish health care system and epidemiological research: From health care contacts to database records. Clin Epidemiol 11, 563-591.

[12] Schmidt M, Pedersen L, Sørensen HT (2014) The Danish Civil Registration System as a tool in epidemiology. Eur $J$ Epidemiol 29, 541-549.

[13] Schmidt M, Schmidt SAJ, Sandegaard JL, Ehrenstein V, Pedersen L, Sørensen HT (2015) The Danish National Patient Registry: A review of content, data quality, and research potential. Clin Epidemiol 7, 449-490. 
[14] Munk-Jørgensen P, Mortensen PB (1997) The Danish Psychiatric Central Register. Danish Med Bull 44, 82-84.

[15] Gjerstorff ML (2011) The Danish Cancer Registry. Scand J Public Health 39, 42-45.

[16] Söhne M, Vink R, Büller HR (2009) Clinical presentation of pulmonary embolism. In Deep Vein Thrombosis and Pulmonary Embolism, Beek EJRv, Bûller HR, Oudkerk M, eds. John Wiley \& Sons, Ltd., UK, pp. 61-69.

[17] Söhne M, Vink R, Büller HR (2009) Clinical presentation of deep vein thrombosis. In Deep Vein Thrombosis and Pulmonary Embolism, Beek EJRv, Büller HR, Oudkerk M, eds. John Wiley \& Sons, Ltd, UK, pp. 53-60.

[18] Charlson ME, Pompei P, Ales KL, MacKenzie CR (1987) A new method of classifying prognostic comorbidity in longitudinal studies: Development and validation. J Chronic Dis 40, 373-383.

[19] Weiss NS (2006) Clinical epidemiology: The study of the outcome of illness, Oxford University Press, New York.

[20] Breslow NE, Day NE (1987) Statistical methods in cancer research. Volume II-The design and analysis of cohort studies. IARC Sci Publ, pp. 1-406.

[21] Iodice S, Gandini S, Löhr M, Lowenfels AB, Maisonneuve $\mathrm{P}$ (2008) Venous thromboembolic events and organ-specific occult cancers: A review and meta-analysis. J Thromb Haemost 6, 781-788.

[22] van der Willik KD, Schagen SB, Ikram MA (2018) Cancer and dementia: Two sides of the same coin? Eur J Clin Invest 48, e13019.

[23] Ganguli M (2015) Cancer and dementia: It's complicated. Alzheimer Dis Assoc Disord 29, 177-182.

[24] Piccioli A, Lensing AW, Prins MH, Falanga A, Scannapieco GL, Ieran M, Cigolini M, Ambrosio GB, Monreal M, Girolami A, Prandoni P (2004) Extensive screening for occult malignant disease in idiopathic venous thromboembolism: A prospective randomized clinical trial. J Thromb Haemost 2, 884-889.
[25] Carrier M, Lazo-Langner A, Shivakumar S, Tagalakis V, Zarychanski R, Solymoss S, Routhier N, Douketis J, Danovitch K, Lee AY, Le Gal G, Wells PS, Corsi DJ, Ramsay T, Coyle D, Chagnon I, Kassam Z, Tao H, Rodger MA (2015) Screening for occult cancer in unprovoked venous thromboembolism. N Engl J Med 373, 697-704.

[26] Robin P, Le Roux PY, Planquette B, Accassat S, Roy PM, Couturaud F, Ghazzar N, Prevot-Bitot N, Couturier O, Delluc A, Sanchez O, Tardy B, Le Gal G, Salaun PY (2016) Limited screening with versus without (18)Ffluorodeoxyglucose PET/CT for occult malignancy in unprovoked venous thromboembolism: An open-label randomised controlled trial. Lancet Oncol 17, 193-199.

[27] Sundbøll J, Adelborg K, Munch T, Frøslev T, Sørensen HT, Bøtker HE, Schmidt M (2016) Positive predictive value of cardiovascular diagnoses in the Danish National Patient Registry: A validation study. BMJ Open 6, e012832.

[28] Storm HH, Michelsen EV, Clemmensen IH, Pihl J (1997) The Danish Cancer Registry-history, content, quality and use. Dan Med Bull 44, 535-539.

[29] Phung TK, Andersen BB, Høgh P, Kessing LV, Mortensen PB, Waldemar G (2007) Validity of dementia diagnoses in the Danish hospital registers. Dement Geriatr Cogn Disord 24, 220-228.

[30] Jones ME, Swerdlow AJ (1998) Bias in the standardized mortality ratio when using general population rates to estimate expected number of deaths. Am J Epidemiol 148, 1012-1017. 